\title{
The long-term effects of methamphetamine exposure during pre-adolescence on depressive-like behaviour in a genetic animal model of depression
}

\author{
Moné Mouton $^{1} \cdot$ Brian H. Harvey ${ }^{1}$ - Marike Cockeran ${ }^{2}$. Christiaan B. Brink ${ }^{1}$
}

Received: 10 July 2015 / Accepted: 11 November 2015 /Published online: 18 November 2015

(C) Springer Science+Business Media New York 2015

\begin{abstract}
Methamphetamine (METH) is a psychostimulant and drug of abuse, commonly used early in life, including in childhood and adolescence. Adverse effects include psychosis, anxiety and mood disorders, as well as increased risk of developing a mental disorder later in life. The current study investigated the long-term effects of chronic METH exposure during pre-adolescence in stress-sensitive Flinders Sensitive Line (FSL) rats (genetic model of depression) and control Flinders Resistant Line (FRL) rats. METH or vehicle control was administered twice daily from post-natal day 19 (PostND19) to PostND34, followed by behavioural testing at either PostND35 (early effects) or long-lasting after withdrawal at PostND60 (early adulthood). Animals were evaluated for depressive-like behaviour, locomotor activity, social interaction and object recognition memory. METH reduced depressive-like behaviour in both FSL and FRL rats at PostND35, but enhanced this behaviour at PostND60. METH also reduced locomotor activity on PostND35 in both FSL and FRL rats, but without effect at PostND60. Furthermore, METH significantly lowered social interaction behaviour (staying together) in both FRL and FSL rats at PostND35 and PostND60, whereas self-grooming time was significantly reduced only at PostND35. METH treatment enhanced exploration of the familiar vs. novel object in the novel object recognition test (nORT) in FSL and FRL rats on
\end{abstract}

\section{Christiaan B. Brink}

tiaan.brink@nwu.ac.za

1 Centre of Excellence for Pharmaceutical Sciences, Faculty of Health Sciences, Division of Pharmacology, North-West University, Internal box 16, Potchefstroom campus, Potchefstroom 2520, Republic of South Africa

2 Research Entity for Medicine Usage in South Africa, Faculty of Health Sciences, North-West University, Potchefstroom 2520, South Africa
PostND35 and PostND60, indicative of reduced cognitive performance. Thus, early-life METH exposure induce social and cognitive deficits. Lastly, early-life exposure to METH may result in acute antidepressant-like effects immediately after chronic exposure, whereas long-term effects after withdrawal are depressogenic. Data also supports a role for genetic predisposition as with FSL rats.

Keywords Methamphetamine · Finders Sensitive Line . Flinders Resistant Line · Depression · Early-life exposure

\section{Introduction}

Methamphetamine (METH), a derivative of amphetamine, is a highly addictive synthetic psychostimulant, mostly abused for recreational purposes. In fact, it has been considered the second most popular illicit drug world-wide (Cruickshank and Dyer 2009), with an estimated excess of 35 million people regularly abusing METH (Vos et al. 2010; Mehrjerdi et al. 2014). Contributing to this growing global epidemic (Cruickshank and Dyer 2009; Yamamoto et al. 2010), METH can be easily synthesized at low cost using house-hold chemicals and over the counter drugs.

Following an initial phase of psychostimulation, METH produces a unique pseudodepressive state due to its ability to directly affect monoamine regulation. Symptoms include anhedonia, fatigue, sleep disturbances, loss of appetite, lack of motivation, irritability and poor concentration, thus relating to many features of major depressive disorder (MDD) (McKetin et al. 2011). Furthermore, early-life exposure to adverse environmental factors, including drug abuse, has been shown to be strongly associated with the development of behavioural abnormalities, neuropsychiatric disease and neurodegenerative disease later in life (Strauss et al. 2014; 
Modgil et al. 2014). Data suggest that continued and repeated exposure to psychostimulants leads to sensitisation of reward and psychomotor effects, most likely resulting from neurobiological changes, particularly in the mesolimbic dopamine (DA) system. These changes are long-lasting, including alterations in gene transcription, RNA and protein synthesis and modifications of neuron morphology (Achat-Mendes et al. 2003).

It has been documented that early-life (pre-adolescent and adolescent) exposure to psychostimulants impairs neural plasticity and is associated with an eventual decrease in the rewarding effect of drug abuse (Achat-Mendes et al. 2003), whereas prenatal exposure to METH results in sustained deficits in attention, long-term spatial and verbal memory as well as visual motor integration during neurocognitive testing (Thompson et al. 2009). An array of evidence thus demonstrates that early life adversity contributes significantly to the susceptibility of adulthood psychopathology, medical illnesses (such as depression), and maladaptive psychosocial functioning (Koehler 2006) as well as altered hippocampal plasticity (Ansorge et al. 2007).

METH also impairs novel location and novel object recognition memory when administered to rats during phases associated with hippocampal development (Siegel et al. 2011). In addition, neuroimaging studies in humans after METH abuse have demonstrated smaller striatum and hippocampus volumes, a decrease in dopamine $\mathrm{D}_{2}$ receptor number and a lower dopamine transporter density (Thompson et al. 2009), similar to what is seen in depressed individuals. Although numerous pre-clinical studies have focused on prenatal exposure to METH and its late-life effects, few studies have focused on the consequences of early-life (pre-adolescent and adolescent) exposure to METH. In fact, there are concerns about the increasing rate of exposure of children to METH (Quigley 2015) and it is thus crucial that the adverse effects during this vulnerable developmental stage are further investigated.

Since METH-induced depression is one of the key symptoms that require treatment during the withdrawal phase as well as later on in life, this study focused on the development of depressive-like behaviour (in an animal model) following chronic (16 days) METH versus vehicle administration during pre-adolescence. Since depression is associated with early life adversity (Modgil et al. 2014), and also since individuals who abuse METH are often from challenging backgrounds, they represent a population already at higher risk for developing a mood disorder. In fact, the combined impact of METH abuse plus early life adversity would fit the paradigm of the double hit hypothesis (Nabeshima and Kim 2013). For this reason, we used the Flinders Sensitive line rat (FSL), a genetic rodent model of depression, to assess whether genetic predisposition plus METH exposure presents a greater risk for developing depressive like behaviour later in life. The effect of METH was assessed using a battery of behavioural tests that have validity for depression, namely learned helplessness, as well as social and cognitive dysfunction.

The working hypothesis of this study was that chronic METH treatment will induce long-term depressive-like behaviours later in life. Moreover, we postulate that combined METH plus genetic predisposition poses a greater risk for developing late-life behavioural anomalies akin to MDD. This study will contribute to our understanding of the neurobehavioural dysfunction brought about by earlylife exposure to METH and in particular how METH abuse may precipitate or worsen depressive-like symptoms in susceptible individuals.

\section{Materials and methods}

\section{Animals}

Male and female Flinders Sensitive Line (FSL) and Flinders Resistant Line (FRL) rats were supplied, housed and bred by the Vivarium at the Pre-Clinical Drug Development Platform (PCDDP) of the North-West University. The original rat colonies were obtained from Dr. David H Overstreet, University of North Carolina, Chapel Hill, North Carolina, USA. The FSL rat line is a well-described, validated genetic animal model of depression, with the FRL rat line as the normal control (Overstreet and Wegener 2013).

The animals were housed 4 rats per cage under conditions of constant temperature $\left(22 \pm 1{ }^{\circ} \mathrm{C}\right)$ and humidity $(50 \pm 10 \%)$ with a 12:12 h light/dark cycle (lights on at 06:00 and lights off at 18:00). Food and water were provided ad libitum. Cages were cleaned and bedding (sawdust) replaced weekly.

All experiments conformed to the guidelines of the South African National Standards: The care and use of animals for scientific purposes (SANS 10386:2008) and were approved in accordance with the regulations set by the AnimCare animal research ethics committee of the North-West University, project ethics approval no. NWU-000,105-11-S5).

\section{Drug treatment}

(+)-Methamphetamine hydrochloride (METH) was purchased from Sigma Aldrich, Missouri, USA (catalogue no. M8750$25 \mathrm{G}$; purity $>98 \%$ ), imported under South African permit no. IMP/626/2011, kept under licence no. POS107/2011/2012 and usage logged as prescribed by national legislation. METH crystals were dissolved in saline (vehicle) for subcutaneous (SC) administration. Male FSL and FRL rats were treated with vehicle or METH twice daily at 09:00 and again at 15:00 from postnatal day nineteen (PostND19) to PostND34 (pre-puberty, with sexual maturity reached around PostND35). It has been argued that due to species differences in neurodevelopmental patterns, it makes sense 
to start animal experiments targeting human adolescents earlier (Spear 2007) when neuronal sprouting is vigorous, hence our choice of age span for treatment. The number of rats per treatment group is indicated on the bar graphs, varying between 10 and 17 per group as a result of varying birth rates in the breeding programme, as well as elimination of few outliers identified with the Grubbs test. METH was administered as before (Strauss et al. 2014) in an escalating dose regimen during the 16 day period (mimicking binging behaviour in humans), with every dose escalating in increments of $0.2 \mathrm{mg} / \mathrm{kg}$ from 0.2 to $6.0 \mathrm{mg} / \mathrm{kg}$ (i.e. $0.2,0.4,0.6, \ldots, 5.6,5.8,6.0,6.0$, $6.0 \mathrm{mg} / \mathrm{kg}$ ), made up to $0.2 \mathrm{ml}$ injection volume. To provide context of the selected dose range, $15 \mathrm{mg} / \mathrm{kg}$ at a $2 \mathrm{~h}$ interval has been used as high dose before (Richards and Yung 2000). Each animal was weighed before injection and the appropriate dose calculated. Saline-treated animals received a fixed dose of $0.2 \mathrm{ml}$ saline SC twice daily. Considering the half-life of METH in rodents being roughly one hour (Rivière et al. 1999), this implies continuous cycles of high systemic levels after dosing and then lower levels after drug clearance in between dosing (typical as with binging in humans), including between the last dose on PostND34 and early testing after short-term clearance on PostND35. Testing on PostND60 would therefore imply total withdrawal and long-lasting effects.

\section{Behavioural testing}

After the 16-day period of drug administration, animals were subjected to a validated battery of behavioural tests (Mokoena et al. 2015), implemented in order of firstly the novel object recognition test (nORT), thereafter the social interaction test (SIT), then the locomotor activity test and finally the forced swim test (FST). These were performed for all treatment groups either early after the injection period on PostND35, or later in life after drug wash-out (withdrawal) in early adulthood on PostND60. Behavioural testing commenced one hour after the start of the dark cycle (i.e. 19:00) when nocturnal animals are active. We demonstrated before that, under our experimental conditions, foregoing tests do not affect the outcome of the subsequent consecutive tests if they are ordered from least to most stressful (Mokoena et al. 2015).

\section{Novel object recognition test}

The well-described novel object recognition test (nORT) was used to evaluate declarative memory in the rats, which is notoriously impaired in patients with depression (Burt et al. 1995). This test relies on the principle that rats prefer to explore a novel (new) object relative to a familiar one, and that recognition of the novel object is dependent on intact memory of the familiar object. The apparatus comprised of a box made of opaque, black Perspex, and with the floor dimensions
$500 \mathrm{~mm} \times 500 \mathrm{~mm}$, and a $400 \mathrm{~mm}$ high surrounding wall, with the arena illuminated with infrared light $(40 \mathrm{~lx})$. Two heavy objects, which cannot be moved by the rat, were placed inside the box.

The habituation trial commenced two days prior to the night of testing when the rats were given the opportunity to explore the empty nORT boxes (one rat per box) for $10 \mathrm{~min}$. Thereafter, on the day of testing, the acquisition trial was conducted. The rats were given the opportunity to explore two identical, steady objects in the nORT box for a period of five minutes. These objects were identical in shape, size and colour and placed $10 \mathrm{~cm}$ from the box walls in two adjacent corners. The rats were then returned to their home cages for a 90 min inter-trial interval (Fischer et al. 2012; Mokoena et al. 2015). The nORT box and objects were cleaned with $10 \%$ ethanol to remove any olfactory cues. The animals were then subjected to the last retention trial, during which they were returned to the test box and presented with two objects, one object identical to the object in the first exploration trial and the second a novel object. The novel object had a different shape, size and colour and was therefore unfamiliar to the rat. Rats were allowed to explore the objects for five minutes, whereafter the rats were removed. Object exploration was defined as sniffing, licking or touching the objects with forepaws (Grayson et al. 2007). Sitting near the object was not considered exploration. The exploration time (seconds) of each object in each trial was scored manually from the recorded videos using two stopwatches, with the scorer blind to the treatment group being scored. With memory intact, rats tend to remember the familiar object and show a tendency to spend more time exploring the novel object. Equal exploration time of the familiar and novel object is regarded as being indicative of impaired memory performance.

\section{Social interaction test}

Social behaviour is known to be deficient in patients with depression (Buck and Siegel 2015). The well-described social interaction test (SIT) was used to measure explorative and social interactive behaviours between pairs of rats in an open field test (OFT) arena and was performed as described before (Mokoena et al. 2015). The apparatus consisted of a black square arena with opaque walls $(100 \mathrm{~cm} \times 100 \mathrm{~cm} \times 40 \mathrm{~cm})$. The floor was divided into $25 \mathrm{~cm} \times 25 \mathrm{~cm}$ equal squares (Sherif and Oreland 1995), with the arena illuminated with infrared light (40 lx). A video camera was placed above the arena to record the activity of the animals for later scoring and analysis.

A pair of unfamiliar rats of the same treatment group was placed together in the arena for a period of $10 \mathrm{~min}$, while the researcher remained outside the testing room during the testing period to avoid distracting the animals. The arena was subsequently cleaned with $10 \%$ ethanol solution between 
tests to eliminate olfactory cues (Ferdman et al. 2007). Social interactive behaviour ("staying together") was scored per pair of rats, whereas self-grooming was scored for each individual rat, with the scorer blind to the treatment group being scored. Less time spent in social interaction and increased selfgrooming was considered indicative of raised anxiety levels.

\section{Locomotor activity}

Spontaneous locomotor activity was accessed using a DigiScan ${ }^{\circledR}$ animal activity monitor (Accuscan Instruments Inc. Columbus, $\mathrm{OH}$, USA), implementing various infrared sensors placed strategically around the box to detect and measure activity within the cage (Cao and Li 2002). Each open field cage had 16 infrared light beams aligned in the horizontal $\mathrm{X}$ and $\mathrm{Y}$ axes, where infrared sensors within the box detect the number of beam breaks caused by animal movement. The rat was placed in the centre of the chamber and the total distance travelled by the animal during a period of five minutes was measured. In this context, locomotor activity was used to distinguish between altered locomotor and psychomotor mobility induced by the drug, as observed in the forced swim test (see above).

\section{Forced swim test}

The well-described forced swim test (FST) was used to assess immobility as measure of depressive-like behaviour of rodents (Blokland et al. 2012; Cryan et al. 2002; Mokoena et al. 2015). The FST does not induce a depressive-like state as such, but has been validated to measure behaviour related to learned helplessness under stress-inducing conditions (Castagné et al. 2009). Importantly, the FSL rat represent a well-described and validated genetic rodent model of depression that displays depressive-like behaviour (e.g. enhanced immobility) without the pre-conditioning swim trial $24 \mathrm{~h}$ prior to the testing swim trial (Overstreet et al. 2005; Overstreet and Wegener 2013).

The rats were individually placed in a water-filled $\left(25^{\circ} \mathrm{C} \pm 2{ }^{\circ} \mathrm{C}\right.$ ) transparent plastic cylinder (height $\times 50 \mathrm{~cm}$; diameter $\times 25 \mathrm{~cm}$; water depth $\times 30 \mathrm{~cm}$ ) from which they cannot escape, under $200 \mathrm{~lx}$ white light, and allowed to swim for a period of $7 \mathrm{~min}$. Video monitoring was used to record the behaviour of the animals, with active scoring undertaken during the mid $5 \mathrm{~min}$, with the scorer blind to the treatment group being scored. Immobility was scored when the animal assumed an immobile posture, floating, and no movements other than to keep their nose or mouths above the water surface to breathe (Blokland et al. 2012).

\section{Statistical analyses}

When comparing only two data points, the Student's $t$ test was used. Normality of the data was determined using the Shapiro-
Wilk test. For multiple comparison of data Nonparametric two-way ANOVA based on ranked data was performed if the assumption of normality was violated. In other instances, the two-way ANOVA was used, and when this analysis indicated interaction between the main factors (i.e. rat line and drug treatment, respectively), it was followed by the Tukey posthoc test. Three-way analyses was not necessary, since the third main factor, namely age at PND35 vs PND60 was not directly compared. GraphPad Prism ${ }^{\circledR}$ version 6 (GraphPad Software, San Diego California USA, www.graphpad.com) was used for statistical analysis and graphical presentations, except for non-parametric statistical analyses when indicated, where IBM SPSS Statistics version 22 was used (IBM Corp. Released 2013. IBM SPSS Statistics for Windows, Version 22.0. Armonk, NY: IBM Corp). A $5 \%$ confidence limit for error was taken as statistically significant $(P<0.05)$.

\section{Results}

\section{The novel object recognition test}

Figure 1 displays the percentage time spent by vehicle- and METH-treated FSL and FRL rats exploring the familiar and novel objects, respectively, in the nORT. Figure $4 \mathrm{a}$ and $\mathrm{b}$ displays these data at PostND35 (i.e. 1 day after the last dose) and Fig. $4 \mathrm{c}$ and $\mathrm{d}$ at PostND60 (i.e. long-lasting effects after washout).

In Fig. 1a the two-way ANOVA of the data $(\mathrm{F}(1,49)=$ $0.5070, P=0.4798$; non-parametric analysis $\chi_{1}^{2}=0.368$, $P=0.544$ ) indicate that there was no statistically significant interaction between line of rat and drug treatment regarding time sent exploring the familiar object on PostND35, thus no post hoc analysis was performed. The effect of line of rat was not significant $(\mathrm{F}(1,49)=0.1228, P=0.7275$; non-parametric analysis $\left.\chi_{1}^{2}=0.109, P=0.741\right)$, whereas the overall effect of METH treatment was statistically significant in both rat strains relative to vehicle controls in the parametric, but not in the non-parametric test $(\mathrm{F}(1,49)=$ 4.167, $P=0.0466$; non-parametric analysis $\chi_{1}^{2}=2.327$, $P=0.127)$. It should be noted in the figure, however, that in the FSL groups METH did not change \% time exploring the novel object, and that such a difference can only be seen in the FRL rats.

In Fig. $1 \mathrm{~b}$ the two-way ANOVA of the data $(\mathrm{F}(1,49)=$ $2.079, P=0.1557$; non-parametric analysis $\chi_{1}^{2}=0.618$, $P=0.432$ ) indicate that there was no statistically significant interaction between line of rat and drug treatment regarding time sent exploring the novel object on PostND35, hence no post hoc analysis was performed. Both the main factor effects of line of rat $(\mathrm{F}(1,49)=0.4922, P=0.4863$; non-parametric analysis $\chi_{1}^{2}=0.850, P=0.357$ ) and drug 
Fig. 1 The effect of vehicle versus chronic methamphetamine (METH) exposure in Flinders Sensitive Line (FSL) and Flinders Resistant Line (FRL) rats on \% time spent exploring the familiar (a \& c) and novel (b \& d) objects, respectively, and also at (a \& b) PostND35 and (c \& d) PostND60, respectively, in the novel object recognition test. Data points represent the mean \pm S.E.M.

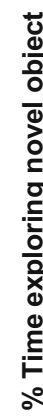
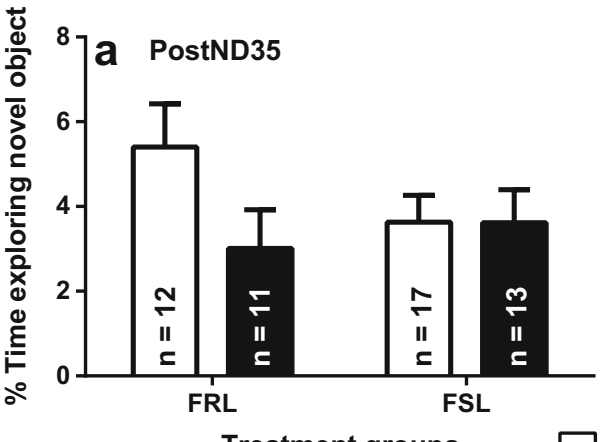

Treatment groups

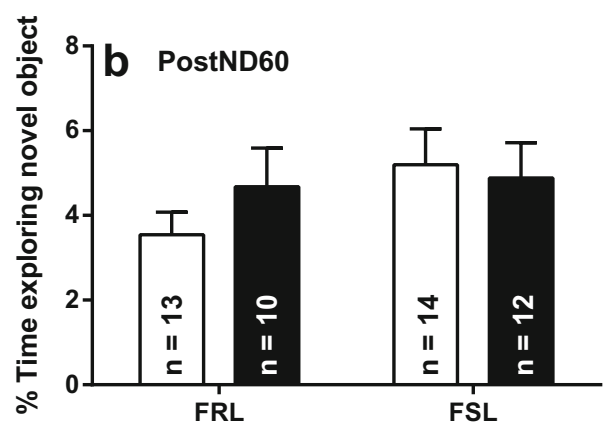

Treatment groups

\section{Vehicle}

METH

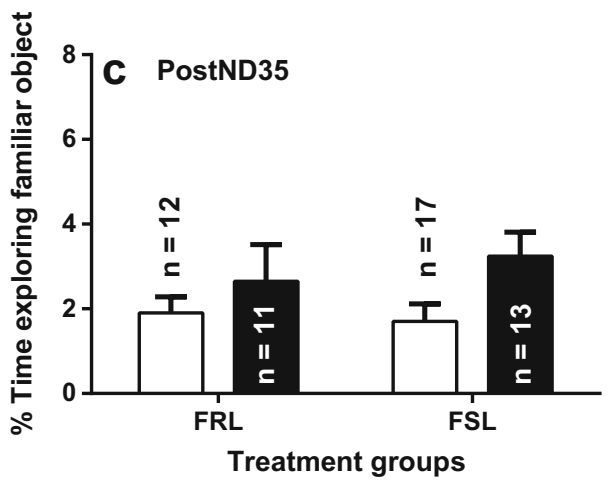

treatment $(\mathrm{F}(1,49)=2.119, P=0.1519$; non-parametric analysis $\left.\chi_{1}^{2}=2.776, P=0.096\right)$ was not statistically significant.

In Fig. 1c the two-way ANOVA of the data $(\mathrm{F}(1,45)=$ $1.498, P=0.2274$; non-parametric analysis $\chi_{1}^{2}=0.415$, $P=0.519$ ) indicate that there was no statistically significant interaction between line of rat and drug treatment regarding time sent exploring the familiar object on PostND60, hence no post hoc analysis was performed. The effect of line of rat was not significant $(\mathrm{F}(1,45)=0.01232, P=0.9121$; non-parametric analysis $\chi_{1}^{2}=0.065, P=0.798$ ), whereas the effect of METH treatment was statistically significant in both rat strains relative to vehicle controls $(\mathrm{F}(1,45)=25.30, P<0.0001$; nonparametric analysis $\left.\chi_{1}^{2}=18.703, P<0.001\right)$.

In Fig. 1d the two-way ANOVA of the data $(\mathrm{F}(1,49)=$ $0.5070, P=0.4798$; non-parametric analysis $\chi_{1}^{2}=0.555$, $P=0.456)$ indicate that there was no statistically significant interaction between line of rat and drug treatment regarding time sent exploring the novel object on PostND60, so that post hoc analysis was not performed. The effect of line of rat was not significant $(\mathrm{F}(1,49)=0.1228, P=0.7275$; non-parametric analysis $\left.\chi_{1}^{2}=1.174, P=0.279\right)$, whereas the effect of METH treatment was statistically significant in both rat strains relative to vehicle controls $(\mathrm{F}(1,49)=4.167, P<0.0466$; nonparametric analysis $\left.\chi_{1}^{2}=0.281, P=0.596\right)$.

It follows from the statistical analysis, and is illustrated in Fig. 1, that METH significantly increased the time spent exploring the familiar object in both FSL and FRL rats relative to saline-treated rats on PostND35 and PostND60. However, no statistical significance was found regarding time spent exploring the novel object.

\section{The social interaction test}

Figure 2 displays the time spent in social interaction (staying together) and self-directed (self-grooming) behaviour in vehicle- and METH-treated FSL and FRL rats in the social interaction test (SIT). Figure 3a and c displays measurements at PostND35 (i.e. 1 day after the last dose) and Fig. 3b and $d$ at PostND60 (i.e. long-lasting effects after washout).

In Fig. 2a the two-way ANOVA of the data $(\mathrm{F}(1,28)=$ $3.568, P=0.0693$ ) indicates that there was no statistically significant interaction between line of rat and drug treatment regarding staying together (social interaction behaviour) on PostND35, hence no post hoc analysis was performed. The effect of line of rat was not significant $F(1,28)=1.350$, $P=0.2552)$, whereas the effect of METH treatment was statistically significant $(\mathrm{F}(1,28)=42.89, P<0.0001)$ on PostND35.

In Fig. $2 \mathrm{~b}$ the two-way ANOVA of the data $(\mathrm{F}(1,25)=$ $3.448, P=0.0751)$ indicated that there was no statistically significant interaction between line of rat and drug treatment regarding staying together (social interaction behaviour) on PostND60, hence no post hoc analysis was performed. The effect of line of rat was not significant $(\mathrm{F}(1,25)=2.239$, 
Fig. 2 The effect of vehicle versus chronic methamphetamine (METH) exposure in Flinders Sensitive Line (FSL) and Flinders Resistant Line (FRL) rats on social interaction and selfdirected behaviour. Time spent in social interactive behaviours (staying together) (a and $\mathbf{b}$ ) and time spent in self-directed (selfgrooming) behaviour (c and d) was evaluated on PostND35 (a \& c) and PostND60 (b \& d). Data points represent the mean \pm S.E.M.
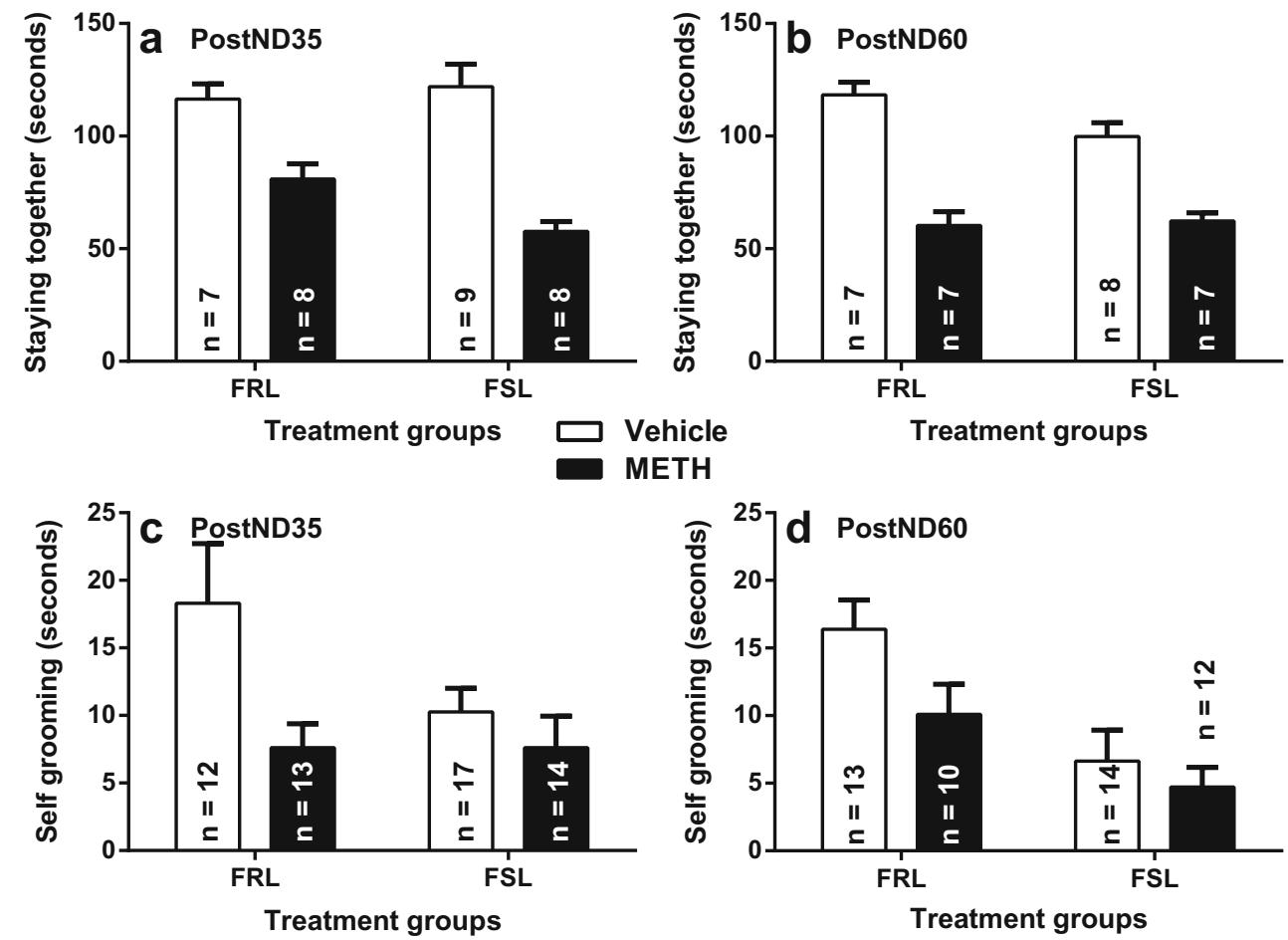

$P=0.1471$, whereas the effect of METH treatment was statistically significantly $(\mathrm{F}(1,25)=75.27, P<0.0001)$ on PostND60.

In Fig. 2c the two-way ANOVA of the data $(\mathrm{F}(1,52)=$ $2.376, P=0.1292$; non-parametric analysis $\chi_{1}^{2}=0.449$, $P=0.503)$ indicated that there was no statistically significant interaction between line of rat and drug treatment regarding self-grooming (self-directed behaviour) on PostND35, so that post hoc analysis is not applicable. The effect of line of rat was also not significant $(\mathrm{F}(1,52)=2.359, P=0.1307$; non-parametric analysis $\left.\chi_{1}^{2}=1.372, P=0.242\right)$, whereas the effect of METH treatment was statistically significant $(\mathrm{F}(1,52)=6.534, P=0.0135$; non-parametric analysis $\left.\chi_{1}^{2}=6.218, P=0.013\right)$ on PostND35.

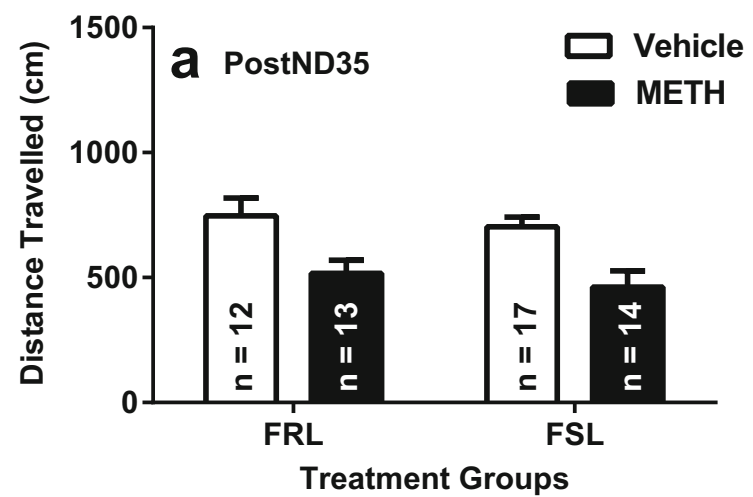

Fig. 3 The effect of vehicle versus chronic methamphetamine (METH) exposure in Flinders Sensitive Line (FSL) and Flinders Resistant Line (FRL) rats on locomotor activity in the Digiscan Apparatus. The total
In Fig. $2 \mathrm{~d}$ the two-way ANOVA of the data $(\mathrm{F}(1,45)=$ $1.088, P=0.3024$; non-parametric analysis $\chi_{1}^{2}=0.834$, $P=0.361)$ indicated that there was no statistically significant interaction between line of rat and drug treatment regarding self-grooming (self-directed behaviour) on PostND60, and therefore again post hoc analysis is not applicable. The effect of line of rat $\mathrm{F}(1,45)=12.94, P=0.0008$; non-parametric analysis $\chi_{1}^{2}=11.860, P=0.001$ ) was statistical significant, whereas the effect of drug treatment was not statistically significant $\mathrm{F}(1,45)=3.807, P=0.0573$; non-parametric analysis $\left.\chi_{1}^{2}=1.931, P=0.165\right)$.

It follows from the statistical analysis, and is illustrated in Fig. 2, that METH significantly lowered the amount of time that both FSL and FRL rats stayed together (social interactive

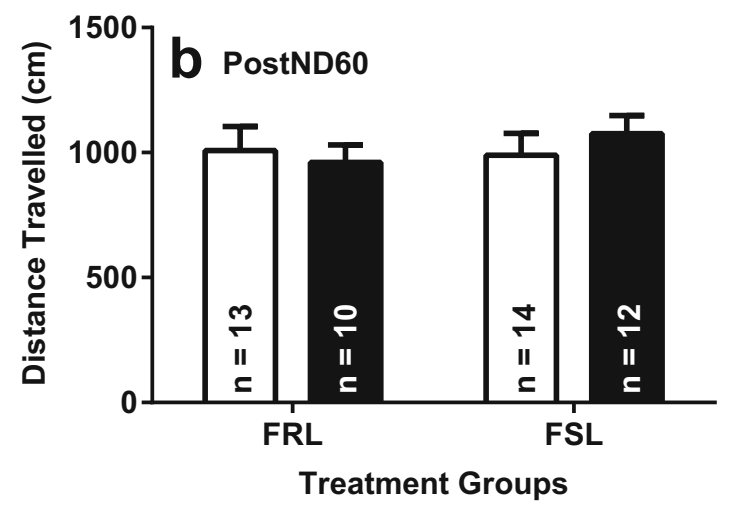

distance travelled was evaluated on PostND35 (a) and PostND60 (b). Data points represent the mean \pm S.E.M. 
behaviour) on PostND35 and PostND60. METH also decreased self-grooming time (self-directed behaviour) in both FSL and FRL rats on PostND35, but not significantly on PostND60.

\section{Locomotor activity test}

Figure 3 displays the total distance travelled by vehicle- and METH-treated FSL and FRL rats, respectively, in the locomotor activity test. Figure 3 a depicts measurements at PostND35 (i.e. 1 day after the last dose) with Fig. 3b at PostND60 (i.e. long-lasting effects after washout).

In Fig. 3a the two-way ANOVA of the data $(\mathrm{F}(1,52)=$ $0.01303, P=0.9095)$ indicates that there was no statistically significant interaction between the line of rats and drug treatment regarding locomotor activity on PND35, hence no post hoc analysis was performed. The effect of line of rat was not statistically significant $\mathrm{F}(1,52)=0.7618, P=0.3868)$, whereas the effect of METH treatment was statistically significant $(\mathrm{F}(1,52)=17.49, P=0.0001)$ on PostND35.

In Fig. $3 \mathrm{~b}$ the two-way ANOVA of the data $\mathrm{F}(1,45)=$ $0.6124, P=0.4380)$ indicated that there was no statistically significant interaction between the line of rats and drug treatment regarding locomotor activity on PND60, hence no post hoc analysis was performed. Both the main factor effects of line of rat $(\mathrm{F}(1,45)=0.3190, P=0.5750)$ and drug treatment $\mathrm{F}(1,45)=0.05259, P=0.8197)$ were not statistically significant.

It follows from the statistical analysis, and is illustrated in Fig. 3, that METH reduced locomotor activity in both FSL and FRL rats on PostND35, but not on PostND60. The effect of METH on locomotor activity was similar in FSL and FRL rats so evidence of a role for genetic predisposition was not observed in this test.

\section{The forced swim test}

Figure 4 displays the immobility time for vehicle- and METHtreated FSL and FRL rats, respectively, in the forced swim test (FST). Figure 4a depicts behaviour at PostND35 (i.e. 1 day after the last dose) and Fig. 4b at PostND60 (i.e. long-lasting effects after washout and complete withdrawal).

In Fig. 4a the two-way ANOVA of the data $(\mathrm{F}(1,49)=$ $0.2335, P=0.6311$; non-parametric analysis $\chi_{1}^{2}=0.205$, $P=0.651)$ indicates that there was no statistically significant interaction between line of rat and drug treatment regarding immobility at PND35, hence no post hoc analysis was performed. Both main factor effects were statistically significant, i.e. the line of rats (FSL vs. FRL) $(\mathrm{F}(1,49)=26.52$, $P<0.0001$; non-parametric analysis $\chi_{1}^{2}=10.022, P=0.002$ ) and drug treatment (METH vs. VEH) $(\mathrm{F}(1,49)=43.28$, $P<0.0001$; non-parametric analysis $\chi_{1}^{2}=10.022, P<0.001$ ).
In Fig. $4 \mathrm{~b}$ the two-way ANOVA of the data $(\mathrm{F}(1,44)=$ 3.954, $P=0.0530$; non-parametric analysis $\chi_{1}^{2}=0.333$, $P=0.564$ ) indicates that there was no statistically significant interaction between line of rat and drug treatment regarding immobility on PND60, hence no post hoc analysis was performed. Both main factor effects were statistically significant, i.e. the line of rats $\mathrm{F}(1,44)=49.41, P<0.0001$; nonparametric analysis $\left.\chi_{1}^{2}=10.464, P=0.001\right)$ and also drug treatment $\mathrm{F}(1,44)=90.39, P<0.0001$; non-parametric analysis $\left.\chi_{1}^{2}=26.233, P<0.001\right)$.

It follows from the statistical analysis, and is illustrated in Fig. 4, that the FSL rats displayed enhanced immobility relative to FRL rats in the FST on PostND35 (before METH withdrawal) and PostND60 (after METH withdrawal). METH decreased immobility at PostND35 (adolescence) in both FRL and FSL rats. However, following 25 days withdrawal on PostND60, METH exerts enhanced immobility in FRL and FSL rats. Therefore, METH withdrawal further augments the genetic predisposition for depressive-like behaviour (immobility) in FSL rats.

\section{Discussion}

Recognition memory, as depicted by novel object recognition (Mathiasen and Dicamillo 2010), was unaltered in FSL vs FRL rats, while METH impaired recognition memory in FRL but not FSL rats PostND35 (Fig. 1a). Meth did not alter recognition memory in either FRL or FSL rats PostND60 (Fig. 1b). Thus, recognition memory remains unaltered in FSL and FRL rats, and is also not significantly affected by METH. Previous studies, however, have reported that animals treated with METH spend less time exploring the novel object compared to their saline controls (Herring et al. 2008; Reichel et al. 2011). Interestingly, memory loss and cognitive deficits, which are symptoms of MDD, were not previously recognised as a behavioural characteristic of the FSL rat (Overstreet et al. 2005). However, more recent studies indicate that cognitive impairment indeed exists in FSL rats (Eriksson et al. 2012), and in particular that of novel object recognition in the FSL rat (Abildgaard et al. 2011). Furthermore, the FSL rat line has been found to represent a suitable model of reversible impairments in emotional processing and memory (Eriksson et al. 2012). The FSL rat also displays predictive validity for evaluation of drugs supporting cognitive performance (Eriksson et al. 2012). It has also been reported that lower doses of METH enhance memory and cognition, and that neurotoxic doses can affect memory and cognition in a different way (Meneses et al. 2011). It is therefore clear that there are conflicting findings in the literature in this regard. However, the current study performed behavioural analyses at PostND35 and PostND60, where previous studies only performed 


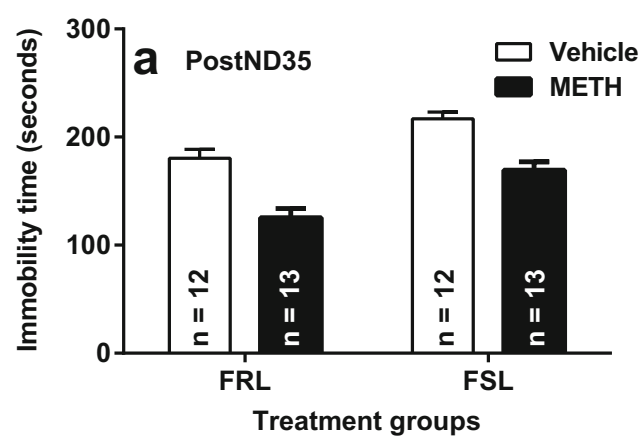

Fig. 4 The effect of vehicle versus chronic methamphetamine (METH) exposure in Flinders Sensitive Line (FSL) and Flinders Resistant Line (FRL) rats on immobility time (seconds) in the forced swim test.

analyses on PostND60, which may explain the difference. This method allowed us to compare the effects of METH directly after the injection period as well as later in life, making the study more robust.

On the other hand, METH enhanced exploration of the familiar object in both rat lines at both PostND35 and PostND60 (Fig. 1c and d). That the rats investigated the familiar object for a greater amount of time relative to controls may be the result of loss of recognition memory for the familiar object. When the rats do not recognize the familiar object, it is interpreted as a deficit in recognition memory as the rats now spend disproportionally more time investigating this object. This lack of recognition memory was found to be longlasting, still present after withdrawal at PostND60 (Fig. 1d). As far as we are aware, this very interesting finding has not been described before, neither is there a clear explanation for this phenomenon. Nevertheless, it is well known that memory involves the laying down of long-term synaptic changes and events related to neuroplasticity that are also involved in neuronal development (Eriksson et al. 2012). Thus, that compromised memory performance, as depicted by enhanced exploration of the familiar object, was observed immediately after METH exposure and after withdrawal is extremely important albeit not unexpected, indicating a sustained adverse effect, putatively via an effect on neurodevelopment.

The data in Fig. 2a and b demonstrate that METH significantly lowers social interaction behaviour (staying together) in both FRL and FSL rats, both shortly following drug treatment (PostND35) and after withdrawal (PostND60). Such behaviour is highly correlated with the clinical features of depression (Buck and Siegel 2015). It is therefore clear that this effect of METH is acute and long-lasting, with the latter putatively related to neurodevelopmental effects that yield sustained adverse neurological manifestations. These findings are in agreement with previously published and unpublished data from our laboratory (Strauss 2012; Strauss et al. 2014). Other previous studies have also shown a decrease in frequency and duration of social interaction between a pair of unfamiliar METH-treated rats (Šlamberová et al. 2010; Strauss

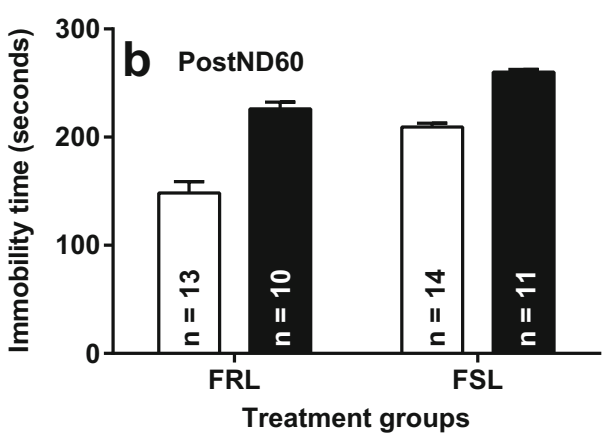

Immobility, as marker of depressive-like behaviour, was evaluated on PostND35 (a) and PostND60 (b). Data points represent the mean \pm S.E.M.

2012; Strauss et al. 2014). However, Samberova and colleagues (2010) found that social interaction was increased after administration of lower doses of METH $(1.25 \mathrm{mg} / \mathrm{kg})$ and decreased after higher doses of METH $(2.5-5 \mathrm{mg} / \mathrm{kg})$ (Šlamberová et al. 2010). Although the aim of METH abuse is typically to experience the "high" and the increased locomotor activity that accompanies it, although the abuse seldom stops there but progresses towards higher and higher doses over time (Stedham 2007). Therefore, the fact that this study used an escalating dose regimen and consequently high doses of the drug are more clinically relevant, and could have contributed to the anti-social behaviour observed. Previous data has also indicated that when METH is administered in higher doses the rats display stereotypical behaviour, for example sitting in the corner of the test box indulging in repetitive head movements with little to no social interaction (Šlamberová et al. 2010). However, true stereotypical behaviour is not necessarily related to depression, unless in the form of an Axis 2 diagnosis. Nevertheless, we do know that METH abusers often present with stereotypic behaviour, while at the same time co-present with depression, as this paper has described.

Differences in social interactive behaviour between FSL and FRL rats did, however, not reach statistical significance in the current study (Fig. 2a and b). Decreased social interactive behaviour is characteristic of the FSL rat, where it displays significant anxiogenic behaviour in the social interaction test, and where pairs of FSL rats tend to avoid each other when passing rather than grooming or crawling over or under each other (Overstreet et al. 2005). The reason for this difference in the current study and that of Overstreet and colleagues is unclear. Interestingly, the unfamiliar environment of the test arena seems to play a vital role in the social interactive behaviour observed. It has been documented that an unfamiliar environment increases time spent in explorative behaviour (locomotion) and therefore decreases the time that the rats stay together. The fact that no habituation trial was conducted in the SIT could have contributed to the decrease in time spent in social interactive behaviour, but then this would apply equally to all treatment groups. 
Self-directed behaviour (self-grooming) was also assessed using the SIT, where METH treatment deceased selfgrooming time significantly on PostND35, but not on PostND60 (Fig. 2c and d). However, on PostND60 FSL rats displayed significantly less self-grooming behaviour than FRL rats, but METH did not make a significant difference. An increase in METH-induced self-directed behaviour was expected, since it has been documented in earlier studies (Strauss 2012), while also elevated self-grooming may be indicative of increased anxiety (Moller et al. 2011). Thus, taking inhibited self-grooming as a measure of anxiety-like behaviour, the current study shows that METH treatment induces anxiety-like behaviour on PostND35, but that this effect is not long-lasting as observed on PostND60.

Locomotor activity assessment (see Fig. 3) indicated that METH treatment decreased locomotor activity in FSL and FRL rats compared to their vehicle controls on PostND35, suggesting that the decreased immobility in the FST (antidepressant-like activity of METH described above) cannot be attributed to enhanced locomotor activity, and hence directly supports an enhanced psychomotor property of the drug under these conditions. However, on PostND60 METH did not affect locomotor activity in FSL or FRL rats compared to their vehicle controls, again confirming that the depressivelike behaviour (enhanced immobility) in the FST relates directly to decreased psychomotor activity and not drug-induced hypolocomotion. It has been shown previously that METHtreated animals are significantly less active (reduced locomotor activity) than their saline-treated controls (Herring et al. 2008; Wallace et al. 1999; Swart 2013). In addition, METHtreated animals display a smaller number of beam breaks than saline treated control animals (Herring et al. 2008), thus similar to what we describe here. However, some earlier work suggest that assessment of repetitive beam breaks does not necessarily reveal METH-induced hyperactivity, although there is evidence for an increase in focused movement, possibly indicative of stereotypy (Herring et al. 2008). Although repetitive beam breaks are not necessarily a measure of stereotypy, they do indicate that aspects of focused movements contribute to the spectrum of stereotypic behaviours observed in METH-treated animals (Herring et al. 2008).

However, after wash-out, we found that animals did not display any significant differences in locomotion regardless of the strain or treatment. Thus METH's ability to decrease locomotor activity seems to be dependent on the immediate effects of METH to alter central monoamine levels (PostND35), as noted above, and is not long-lasting after withdrawal (PostND60). Previous studies have indicated that METH markedly increases locomotor activity initially, with a long-lasting enhancement of locomotor activity over a couple of hours (Wallace et al. 1999) that was lost after metabolism of the drug (Wallace et al. 1999). Also, the latter authors noted that METH-treated animals exhibited lower activity during the initial exploratory phases (for the first $30 \mathrm{~min}$ ) than saline-treated rats, although locomotor activity in both groups were comparable during the last $30 \mathrm{~min}$ of the trial. Thus both METH- and saline-treated animals showed similar levels of activity at the end of the test period (Wallace et al. 1999). The current study is the first to investigate long-lasting effects, after 25 days of withdrawal.

The data obtained from the FST (Fig. 4) indicate that FSL rats were as a group more immobile than FRL rats, confirming the face validity of the FSL genetic rat model of depression under our experimental conditions. Furthermore, chronic pre-puberty administration of METH to FSL and FRL rats for 16 days induced antidepressant-like behaviour when assessed early after the last day of treatment (PostND35), suggesting that METH is initially antidepressant in nature. Other studies have similarly shown that METH causes a decrease in immobility time in a dose dependant manner when tested directly after administration (Shimazu et al. 2005). This anti-depressant response observed at PostND35, however, was evident only in the FST, whereas behaviour in the SIT and nORT, as described below, would be more suggestive of depressogenic-like effects. After 25 days withdrawal (PostND60) a depressogenic effect was observed in METHtreated FRL and FSL rats relative to vehicle-treated animals in the FST. In fact, immobility observed in METH-treated FRL rats closely resembled that of METH-naïve FSL rats, and ultimately immobility was the largest in METH-treated FSL rats (see a possible role for genetic susceptibility below). It may be deduced that pre-puberty exposure of rats to METH results in an immediate antidepressant-like effect, while long-lasting depressogenic effects occur after prolonged discontinuation after chronic exposure, putatively via effects on neurodevelopment and a resulting effect on regional brain monoamines, as described recently (Strauss et al. 2014). Previous studies have indicated that withdrawal from chronic amphetamine administration can result in behavioural alterations with characteristics similar to that of depression in humans (McGregor et al. 2003), for example the elevation of the reward threshold in the brain with consequent deficits in reward signalling (Cryan et al. 2002). The current study suggests a psychostimulant withdrawal-induced depression, in line with these earlier findings. This is a robust phenomenon that is detectable in numerous behavioural tests in rats, including the intracranial self-stimulation (ICSS) procedure, the FST as well as the tail suspension test (Cryan et al. 2002). Psychostimulant withdrawal-induced depression also seems to be long-lasting, as seen with ecstasy (McGregor et al. 2003), and may be the result of the enduring neurotoxic effects of METH (McGregor et al. 2003). In fact, in agreement with numerous reports that METH causes neuronal damage via increased oxidative stress (Tocharus et al. 2010), a recent study in our laboratory demonstrated that chronic METH exposure induces pronounced 
perturbations in regional brain monoamines in parallel with a significant decrease in superoxide dismutase (SOD) activity and increase in lipid peroxidation in various brain regions of the rat (Strauss 2012).

Contrary to the antidepressant-like effect described immediately post treatment, the long-lasting developmental effect of post-METH withdrawal was also additive to the congenital depressive-like phenotype in FSL rats, inducing a profound depressogenic action. The presence of a role for genetic susceptibility in this response supports the two-hit hypothesis of depression, suggesting that individuals that already have a genetic predisposition to depression (1st hit) may be more susceptible to developing depression when abusing METH early in life (2nd hit). This finding is also supported by similar unpublished data from our laboratory using FSL rats (Swart 2013). On the other hand, studies using combined METH plus social isolation rearing (SIR) (Strauss et al. 2014), thus a history of environmental adversity, did not support the two hit hypothesis. However, Strauss argues in favour of a ceiling effect that prevented more prominent neurobehavioral deficits from occurring (Strauss et al. 2014). In yet another study of METH administration, maternal separation and the combination of the two stressors decreased BDNF levels in both the dorsal and ventral hippocampus. Nerve growth factor (NGF) remained unaltered by either stressor alone or in combination in the dorsal hippocampus. Importantly, however, in the ventral hippocampus associated with anxiety-related responses, maternal separation decreased NGF, but this was reversed by methamphetamine via a compensatory mechanism (Dimatelis et al. 2014). These data underline the complexity of brain response and compensatory mechanisms involved. That METH plus FSL phenotype supports the two-hit hypothesis, whereas METH plus either maternal separation or social isolation does not, is therefore of interest. We postulate that METH fails to illicit a compensatory mechanism in FSL rats as it apparently does in the aforementioned two models and that the FSL plus METH model is arguably more suitable for studying the two-hit hypothesis. However, further studies that investigate this phenomenon are needed.

\section{Summary and conclusion}

The current study demonstrates that chronic METH treatment produces significant behavioural effects, of which some are observed early after the injection period (PostND35) and others in the long-term after METH withdrawal, manifesting in early adulthood (PostND60). Importantly, chronic early-life exposure to METH induces antidepressant-like behaviour before withdrawal, but this is reversed after withdrawal into early adulthood, suggesting METH-induced long-term depressogenic effects in genetically susceptible and control animals. This long-lasting effect also seems to be additive to the congenital depressive-like phenotype of FSL rats, suggesting a role for genetic predisposition. This observation would be in line with a two-hit hypothesis of depression, suggesting that the manifestation of depression will result when genetic predisposition is followed by an environmental stressor (i.e. METH) later in life. The data suggest as working hypothesis that juvenile individuals with a predisposition to depression early in life and abusing METH, may be more susceptible to developing depression later in life. Locomotor data further confirmed that the immobility in the FST indeed reflects psychomotor effects, which cannot be explained by locomotor effects. The study also demonstrated that METH significantly lowers social interaction behaviour in both FRL and FSL rats, both soon following drug treatment (PostND35) and after withdrawal (PostND60). In addition the rats investigated the familiar object for a greater amount of time in the nORT on PostND35 and PostND60, indicative of impaired recognition memory of the familiar object. These data confirm that METH results in deficits in social interaction and in cognitive memory, probably due to sustained adverse neurodevelopmental effects.

Thus, early-life exposure to adversity, like drug abuse, may aid the development of late-life behavioural abnormalities, in particular depression. The current study demonstrated that pre-adolescent exposure to METH can reproduce many of the behavioural changes seen in depressed individuals. Furthermore, although further study is required to delineate the specific neurodevelopmental mechanisms involved as well as potential treatment options, the current data suggests that early-life exposure to METH in predisposing individuals (male FSL rats) may represent a useful two-hit model with which to study early life adversity on behavioural abnormalities later in life, particularly mood disorders.

Prospective studies should investigate long-lasting neurobiological effects of METH, in particular involving dopaminergic and serotonergic neurotransmission. Biomarkers of neuroinflammation, such as the cytokines or the kynurenine pathway, and of neuroplasticity, such as brain-derived neurotrophic factor, should also be assessed. Of particular importance would be to assess resilience following early-life METH-induced adversity, for example evaluating behavioural and neurological stress-response. Lastly, it would be crucial to evaluate these METH-induced changes and long-lasting effects following pre-natal exposure to $\mathrm{METH}$, or exposure during puberty.

Acknowledgments This study was supported by a grant from the South Africa Medical Research Council.

Initial protocol development was done by Laetitia Swart as part of a Master's study at the North-West University, and the work presented as a poster at the 46th Annual Congress of the South African Society for Basic and Clinical Pharmacology 2012, South Africa.

The authors would like to thank Dr. Suria Ellis for statistical consultation. The authors would also like to acknowledge Cor Bester, Antoinette Fick, and Hylton Buntting for overseeing the welfare of the animals. 
Compliance with ethical standards All applicable international, national, and/or institutional guidelines for the care and use of animals were followed. All procedures performed in studies involving animals were in accordance with the ethical standards of the institution or practice at which the studies were conducted. Details about ethical clearance is provided under "Materials and Methods"/“Animals" above.

This article does not contain any studies with human participants performed by any of the authors.

\section{References}

Abildgaard A, Solskov L, Volke V, Harvey BH, Lund S, Wegener G (2011) High-fat diet exacerbates depressive-like behaviour in the Flinders Sensitive Line (FSL) rat, a genetic model of depression. Psychoneuroendocrinology 36:623-633. doi:10.1016/j.psyneuen. 2010.09.004

Achat-Mendes C, Anderson KL, Itzhak Y (2003) Methylphenidate and MDMA adolescent exposure in mice: Long-lasting consequences on cocaine-induced reward and psychomotor stimulation in adulthood. Neuropharmacology 45:106-115. doi:10.1016/S00283908(03)00135-7

Ansorge MS, Hen R, Gingrich JA (2007) Neurodevelopmental origins of depressive disorders. Curr Opin Pharmacol 7:8-17. doi:10.1016/j. coph.2006.11.006

Blokland A, Ten Oever S, van Gorp D, van Draanen M, Schmidt T, Nguyen E, Krugliak A, Napolentano A, Keuter S (2012) Klinkenberg I (2012) The use of a test battery assessing affective behavior in rats: Order effects. Behav Brain Res 228:16-21. doi:10.1016/j.bbr.2011.11.042

Buck JM, Siegel JA (2015) The effects of adolescent methamphetamine exposure. Front Neurosci 9(151):1-5. doi:10.3389/fnins.2015. 00151

Burt DB, Zembar MJ, Niederehe G (1995) Depression and memory impairment: a meta-analysis of the association, its pattern, and specificity. Psychol Bull 117(2):285-305 http://dx.doi.org/10.1037/00332909.117.2.285

Cao B, Li Y (2002) Reduced anxiety- and depression-like behaviours in emx 1 homozygous mutant mice. Brain Res 937:32-40. doi:10. 1016/S0006-8993(02)02461-7

Castagné V, Moser P, Porsolt RD (2009) Methods of Behavior Analysis in Neuroscience. Chapter 6: Behavioral Assessment of Antidepressant Activity in Rodents. Taylor and Francis Group, LLC. 2nd edition. 6.1-6.6

Cruickshank CC, Dyer KR (2009) A review of the clinical pharmacology of methamphetamine. Addiction 104(7):1085-1099. doi:10.1111/j. 1360-0443.2009.02564.x

Cryan JF, Markou A, Lucki I (2002) Assessing antidepressant activity in rodents: Recent developments and future needs. Trends Pharmacol Sci 23(5):238-245. doi:10.1016/S0165-6147(02)02017-5

Dimatelis JJ, Russell VA, Stein DJ, Daniels WM (2014) Methamphetamine reversed maternal separation-induced decrease in nerve growth factor in the ventral hippocampus. Metab Brain Dis 29:433-439. doi:10. 1007/s11011-014-9481-Z

Eriksson TM, Delagrange P, Spedding M, Popoli M, Mathé AA, Ogren SO, Svenningsson P (2012) Emotional memory impairments in a genetic rat model of depression: involvement of 5-HT/MEK/Arc signaling in restoration. Mol Psychiatry 17:173-184. doi:10.1038/ mp. 2010.131

Ferdman N, Murmu RP, Bock J, Braun K, Leshem M (2007) Weaning age, social isolation, and gender, interact to determine adult explorative and social behavior, and dendritic and spine morphology in prefrontal cortex of rats. Behav Brain Res 180(2): 174-182. doi:10.1016/j.bbr.2007.03.011
Fischer CW, Liebenberg N, Elfving B, Lund S, Wegener G (2012) Isolation-induced behavioural changes in a genetic animal model of depression. Behav Brain Res 230(1):85-91. doi:10.1016/j.bbr. 2012.01.050

Grayson B, Idris NF, Neill JC (2007) Atypical antipsychotics attenuate a sub-chronic PCP-induced cognitive deficit in the novel object recognition task in the rat. Behav Brain Res 184(1):31-38. doi:10. 1016/j.bbr.2007.06.012

Herring NR, Schaefer TL, Gudelsky GA, Vorhees CV, Williams MT (2008) Effect of (+)-Methamphetamine on Path Integration Learning, Novel Object Recognition, and Neurotoxicity in Rats. Psychopharmacology 199(4):637-650. doi:10.1007/s00213-008$1183-\mathrm{y}$

Koehler B (2006) Neurodevelopment and Depression/Stress. Available from: http://www.isps-us.org/koehler/neurodevelopment.htm. Accessed 26 Oct 2014

Mathiasen JR, Dicamillo A (2010) Novel object recognition in the rat: A facile assay for cognitive function. Curr Protoc Pharmacol 49: 5.59.1-5.59.15. doi:10.1002/0471141755.ph0559s49

McGregor IS, Gurtman CG, Morley KC, Clemens KJ, Blokland A, Li KM, Cornish JL, Hunt GE (2003) Increased anxiety and "depressive" symptoms months after MDMA ("ecstasy") in rats: druginduced hyperthermia does not predict long-term outcomes. Psychopharmacology 168:465-474. doi:10.1007/s00213-003$1452-8$

McKetin R, Lubman DI, Lee NM, Ross JE, Slade TM (2011) Major Depression among methamphetamine users entering drug treatment programs. Med J Aust 195(3):S51-S55

Mehrjerdi ZA, Abarashi Z, Noroozi A, Arshad L, Zarghami M (2014) Correlates of shared methamphetamine injection among methamphetamine injecting treatment seekers: the first report from Iran. Int J STD AIDS 25(6):420-427. doi:10.1177/0956462413512806

Meneses A, Ponce-Lopez T, Tellez R, Gonzalez R, Castillo C, Gasbarri A (2011) Effects of D-amphetamine on short- and long-term memory in spontaneously hypertensive, Wistar-Kyoto and Sprague-Dawley rats. Behav Brain Res 216:472-476. doi: 10.1016/j.bbr.2010.08.035

Modgil S, Lahiri DK, Sharma VL, Anand A (2014) Role of early life exposure and environment on neurodegeneration: implication on brain disorders. Transl Neurosci 3:9

Mokoena ML, Harvey BH, Viljoen F, Ellis SM, Brink CB (2015) Ozone exposure of Flinders Sensitive Line rats is a rodent translational model of neurobiological oxidative stress with relevance for depression and antidepressant response. Psychopharmacology (Epub). doi: 10.1007/s00213-015-3928-8

Moller M, Du Preez JL, Emsley R, Harvey BH (2011) Isolation rearinginduced deficits in sensorimotor gating and social interaction in rats are related to cortico-striatal oxidative stress, and reversed by subchronic clozapine administration. Eur Neuropsychopharmacol 21: 471-483. doi:10.1016/j.euroneuro.2010.09.006

Nabeshima T, Kim H (2013) Involvement of Genetic and Environmental Factors in the Onset of Depression. Exp Neurol 22(4):235-243 http://dx.doi.org/10.5607/en.2013.22.4.235

Overstreet DH, Wegener G (2013) The flinders sensitive line rat model of depression-25 years and still producing. Pharmacol Rev 65(1):143155. doi:10.1124/pr.111.005397

Overstreet DH, Friedman E, Mathé AA, Yadid G (2005) The Flinders Sensitive Line rat: a selectively bred putative animal model of depression. Neurosci Biobehav Rev 29:739-759. doi:10.1016/j. neubiorev.2005.03.015

Quigley R (2015) The innocent 'meth heads': How more and more children are becoming victims of deadly drug as number of 'shake and bake' at-home labs skyrocket. Daily mail (Accessed online at http://www.dailymail.co.uk/news/article-2376753/Theinnocent-meth-heads-Children-victims-deadly-drug-number-homelabs-skyrocket.html on 12 Oct 2015 
Reichel CM, Schwendt M, McGinty JF, Olive MF, See RE (2011) Loss of Object Recognition Memory Produced by Extended Access to Methamphetamine Self-Administration is Reversed by Positive Allosteric Modulation of Metabotropic Glutamate Receptor 5. Neuropsychopharmacology 36:782-792. doi:10.1038/npp.2010.212

Richards JB, Yung K (2000) The effects of high-dose methamphetamine in the aging rat: differential reinforcement of low-rate 72-s schedule behavior and neurochemistry. J Pharmacol Exp Ther 294(3): $850-863$

Rivière GJ, Byrnes KA, Gentry WB, Owens SM (1999) Spontaneous locomotor activity and pharmacokinetics of intravenous methamphetamine and its metabolite amphetamine in the rat. J Pharmacol Exp Ther 291(3):1220-1226

Sherif F, Oreland L (1995) Effect of the GABA-transaminase inhibitor vigabatrin on exploratory behaviour in socially isolated rats. Behav Brain Res 72(1-2):135-140

Shimazu S, Minami A, Kusumoto H, Yoneda F (2005) Antidepressant-like effects of selegiline in the forced swim test. Eur Neuropsychopharmacol 15:563-571. doi:10.1016/j.euroneuro.2005.02.003

Siegel JA, Park BS, Raber J (2011) Methamphetamine exposure during brain development alters the brain acetylcholine system in adolescent mice. J Neurochem 19:89-99. doi:10.1111/j.1471-4159.2011. 07418.x

Šlamberová R, Mikulecká A, Pometlová M, Schutová B, Hrubá L, Deykun K (2010) The effects of methamphetamine on social interaction of adult male rats. Behav Brain Res 214:423-427. doi:10. 1016/j.bbr.2010.06.019

Spear LP (2007) Assessment of adolescent neurotoxicity: Rationale and methodological considerations. Neurotoxicol Teratol 29:1-9

Stedham CW (2007) Methamphetamine abuse: exploring the source of product, income and population of residence in relationship to the age of first use in Minnesota. ProQuest Information and Learning Company, Minnesota: Capella University. (Thesis - PhD)

Strauss L (2012) Effects of chronic methamphetamine exposure during early or late phase development in normal and social isolation reared rats. Potchefstroom: NWU. (Dissertation - MSc)

Strauss L, Brink CB, Moller M, Stein DJ, Harvey BH (2014) LateLife Effects of Chronic Methamphetamine Exposure during Puberty on Behaviour and Corticostriatal Mono-Amines in Social Isolation-Reared Rats. Dev Neurosci 36:18-28. doi:10. $1159 / 000357495$

Swart C (2013) Effects of early-life administration of methamphetamine on depressive-like behaviour later in life in stress-sensitive and control rats. Potchefstroom: NWU. (Dissertation - MSc)

Thompson BL, Levitt P, Stanwood GD (2009) Prenatal exposure to drugs: effects on brain development and implications for policy and education. Nat Rev Neurosci 10(4):303-312. doi:10.1038/ nrn2598

Tocharus J, Khonthun C, Chongthammakun S, Govitrapong P (2010) Melatonin attenuates methamphetamine-induced overexpression of pro-inflammatory cytokines in microglial cell lines. J Pineal Res 48: 347-352. doi:10.1111/j.1600-079X.2010.00761.x

Vos PJ, Cloete KJ, Le Roux A, Kidd M, Jordaan GP (2010) A retrospective review of the trends and clinical characteristics of methamphetaminerelated acute psychiatric admissions in a South African context. Afr J Psychiatry 13(5):390-394

Wallace TL, Gudelsky GA, Vorhees CV (1999) MethamphetamineInduced Neurotoxicity Alters Locomotor Activity, Stereotypic Behavior, and Stimulated Dopamine Release in the Rat. J Neurosci 19(20):9141-9148

Yamamoto BK, Moszczynska A, Gudelsky GA (2010) Amphetamine toxicities: Classical and emerging mechanisms. Ann N Y Acad Sci 1187:101-121. doi:10.1111/j.1749-6632.2009.05141.x 